\title{
Sharing Personal Information is Discounted as a Function of Social Distance
}

\author{
Paul Romanowich ${ }^{1}$ iD \\ Accepted: 7 October 2021 / Published online: 18 November 2021 \\ (c) The Author(s) 2021
}

\begin{abstract}
Social discounting researchers have repeatedly shown that individuals discount sharing the amount of a monetary reward as a function of social distance, and that increasing the available monetary reward decreases sharing. However, no previous study has tested whether sharing nonmonetary commodities are discounted as a function of social distance. The current study tested whether sharing personal information would be discounted similarly to monetary rewards, as well as whether a magnitude effect occurred at a relatively small magnitude difference with 96 university students. A within-participant procedure showed that sharing personal information was discounted as a function of social distance, albeit with a steeper discounting rate relative to both monetary reward magnitudes. However, there was no significant association between personal information discounting rates and monetary discounting rates at either magnitude, suggesting that participants treated each commodity differently (i.e., commodity effect). Replicating previous non-U.S. samples, discounting rates for both monetary reward magnitudes were significantly positively associated with each other and showed a significant magnitude effect, with participants showing significantly steeper discounting rates for the relatively larger monetary rewards. The results for sharing personal information are important because many scams now target personal information in addition to money. Future research should examine what type of personal information is most likely to be shared as a function of social distance, and whether those participants who choose to share more personal information also are at greater risk for scams targeting personal information.
\end{abstract}

Keywords altruism $\cdot$ scams $\cdot$ selfishness $\cdot$ social discounting $\cdot$ vulnerability

\section{Introduction}

Sharing is an important aspect of being human. We can survive and thrive due to our capacity to both cooperate and share with each other (Matsumoto \& Juang, 2017). One research area on sharing, social discounting, has focused on with whom and how much we share as social distance increases (Buddiga \& Locey, 2021; Jones \& Rachlin, 2006). In a typical social discounting task, individuals are asked to either keep a larger amount of money for themselves (e.g., $\$ 155)$, or evenly split a smaller amount with another person at a given social distance (e.g., $\$ 75$ for yourself and $\$ 75$ for the other person). Social distance is how close we psychologically feel to another individual. Social discounting

Paul Romanowich

romanowich@gonzaga.edu

1 Psychology Department, Gonzaga University, 502 East Boone Avenue, Spokane, WA 99258, USA researchers have consistently shown that sharing money decreases hyperbolically as a function of increasing social distance for individuals from many different cultures (Stegall et al., 2019). That is, we share a lot with the people we are closest to, but much less as social distance increases. In addition, the amount available to be shared systematically influences how much is shared; as amount magnitude increases, less is shared (Osinksi, 2010; Rachlin \& Jones, 2008; Romanowich \& Igaki, 2017).

However, sharing is not always beneficial. Criminals now target individuals through scams, either online (Martens et al., 2019) or over the phone. In addition to money, scams can use our propensity to share personal information with another individual (e.g., bank account number, passwords), that can then be used to extract more money by the scammer either directly, or through a third party (Rosoff \& Pontell, 2011). For example, a popular phone-based scam in Japan (ore ore sagi) involves pretending to be a close relative in trouble (e.g., from a traffic accident) who requests cash or 
access to bank information immediately (Brasor, 2019). Although no research connecting social discounting and scams currently exists, previous social discounting research suggests that imitating someone at a close social distance to the caller would increase the probability that the scam will be successful for "sharing" money.

But what about personal information that can be used to access bank accounts or sold to third parties? Perhaps individuals are more hesitant to share this information, relative to money. However, no previous social discounting study has measured whether anything other than money is shared as a function of social distance. Delay discounting, a putative measure for impulsivity also shows a hyperbolic discounting function whereby commodity value decreases as a function of time (Mazur, 1987). Unlike social discounting, there is a large literature showing that different commodities show different discounting properties (Weatherly et al., 2010). For example, Charlton and Fantino (2008) showed that metabolic processing could profitably discriminate between delay discounting rate differences across food, money, music access, video access, and book access. In this case, commodities that serve a direct metabolic function (e.g., food) were discounted at higher rates (i.e., lose value quicker), relative to those less related to metabolism. A recent meta-analysis demonstrated that this commodity effect phenomenon was highly reliable for delay discounting, and that even when discounting for nonmonetary commodities was steeper than monetary commodities, there was a significant relationship between commodities (Odum et al., 2020). That is, individuals that steeply discounted nonmonetary commodities were more likely to also steeply discount money. However, because there is currently no research on whether social discounting shows a commodity effect, the first aim was to determine whether individuals also share personal information like hypothetical money as social distance increases.

Our second aim was to replicate the magnitude effect previously described using a within-participant procedure with participants from the United States. Rachlin and Jones (2008) first reported decreasing sharing as a function of increasing hypothetical money using three magnitudes $(\$ 7.50, \$ 75$, and \$75,000) and four social distances $(1,10$, 50, and 100) in a between-participants design with U.S. participants. ${ }^{1}$ Osinksi (2010) replicated this result using a within-participant procedure with two magnitudes (Polish zlotny [PLN] 494 and 49,400; US \$225 and \$22,500) and two social distances (close and distant) with Polish

\footnotetext{
${ }^{1}$ Rachlin and Jones (2008) tested whether the slope of the discounting rates across the three magnitudes was different from zero, which it was. However, there was no follow-up statistical test between individual magnitudes (e.g., $\$ 7.50$ vs. $\$ 75$ ) to determine which magnitudes were different. Thus, it is unknown if there was a magnitude effect at smaller magnitudes.
}

participants. However, Romanowich and Igaki (2017) only replicated this social discounting magnitude effect with Japanese participants, but not with participants from the United States. In this between-participant design, two magnitudes were used (\$75 and \$750) with seven social distances $(1,2$, $5,10,20,50$, and 100) for both U.S. and Japanese participants (U.S. dollars converted to Japanese yen). Thus, except for Japanese participants, social discounting magnitude effects (e.g., less sharing as magnitude increases) have only been observed with large magnitude shifts (100x or more). U.S. participants may only show a social discounting magnitude effect with a large magnitude shift. A within-participant design, where each participant experiences each magnitude level, has increased statistical power, and increased external validity for economic choices (Charness et al., 2012) when testing whether U.S. participants are sensitive to smaller magnitude shifts (10x).

In the current study, participants completed three social discounting tasks (one for personal information; two monetary magnitudes) to determine (1) whether individuals discount personal information as a function of social distance, and (2) whether a social discounting magnitude effect exists for U.S. participants at smaller magnitude differences. Consistent with delay discounting research (i.e., Odum et al., 2020), we hypothesized that individuals would discount both hypothetical money and personal information in a similar way. That is, there would be a significant correlation between social discounting rates for personal information and money. Although only one previous study has explicitly examined the magnitude effect at smaller magnitude differences with U.S. participants (Romanowich \& Igaki, 2017) with negative results, we hypothesized that there would be a social discounting magnitude effect at a small magnitude difference based on previous studies generally showing this effect. In this case, the social discounting magnitude effect is where individuals share less as monetary magnitude increases.

\section{Methods}

\section{Participants and Procedure}

Ninety-six undergraduate students at a small private university in the northwestern United States who were at least 18 years old and enrolled in an introduction to psychology course during the fall 2020 semester were enrolled in the current study. Participants were recruited through a closed internet-based participant pool and earned $30 \mathrm{~min}$ toward their compulsory experimental research requirement. Institutional review board (IRB) approval was granted before participant solicitation and data collection. All participants consented to the study protocol before study initiation. 
All participants completed four measures in the following order: two monetary social discounting questionnaires (standard and large magnitude), a personal information social discounting task, and a demographic questionnaire. Monetary social discounting magnitude order was randomized between participants. All measures were administered online via the Qualtrics questionnaire platform. Participants completed all measures at their leisure on their personal device (computer or smartphone) within a 48-hr period of signing up for the experiment.

\section{Measures}

\section{Monetary Social Discounting Tasks}

Similar to Jones and Rachlin (2006), participants were asked to imagine (but not write down) a list of 100 people closest to them in the world. Participants were then asked to make choices between a hypothetical monetary amount to keep for themselves (e.g., \$155 for standard magnitude; $\$ 1,550$ for large magnitude), and a hypothetical monetary amount to split evenly between themselves and the other person at a given social distance (e.g., \$75 for each person in standard magnitude; $\$ 750$ for large magnitude). Social discounting was assessed at seven social distances in ascending order for each magnitude: $1,2,5,10,20,50$, and 100. Up to nine choices were made at each social distance for each magnitude. Choices at each social distance began with the largest hypothetical amount for the participant $=\$ 155(\$ 1,550$ large magnitude) alone and decreased by $\$ 10$ (\$100) for each nonsharing choice to a minimum of $\$ 75$ (\$750). The alternative sharing choice was constant at $\$ 75$ (\$750) for each person. The point at which a participant switched from choosing the nonsharing choice to the sharing choice ended all choices for that given social distance and was considered the indifference point. To equate total social discounting task time across participants, a 2-s delay was instituted for each nonsharing choice that was skipped by choosing the sharing choice. For example, if a participant chose the sharing choice (\$75 for both) versus keeping $\$ 125$ (and had already chose keeping \$135, \$145, and \$155 alone), there would be a 10-s delay (five skipped choices at $2 \mathrm{~s}$ each) before the participant could make choices at the next social distance. In this case, the indifference point for that social distance would be $\$ 55$, because the participant gave up $\$ 125$ to receive $\$ 75$ $(\$ 125-\$ 75=\$ 50)$ and allocate $\$ 75$ to the other person, but not $\$ 135(\$ 135-\$ 75=\$ 60)$. Thus, the mean $(\$ 50+\$ 60 / 2)$ was an indifference point equal to $\$ 55$.

\section{Personal Information Social Discounting Task}

Discounting rates for personal information were measured similarly to social discounting. Participants were asked to imagine a list of 50 people closest to them. Participants were then asked to make choices between sharing a certain percentage (e.g., 100\%) of their personal information with the person at a given social distance, or sharing less than that percentage (e.g., $<100 \%$ ) of their personal information. Personal information was specified as "passwords, PIN number, and/or sensitive health information" at the beginning of each social distance (six times total). For each "less than a certain percentage" choice, the next choice decreased by $10 \%$ for personal information shared down to $0 \%$ ("Share none of my personal information with person $x$ on the list"). Thus, participants specified how much personal information they were willing to share with 10 choices in $10 \%$ intervals between $100 \%-0 \%$ for the six social distances $(1,2,5,10$, 20, and 50), akin to a Guttman scale (Coombs et al., 1978). Like the social discounting task, total time engaged in the personal information discounting task was controlled with a 2-s delay for each "less than" choice that was skipped by choosing a specific percentage. Social discounting for personal information was only measured up to social distance 50 because this was the first attempt to measure this commodity for social discounting, and discounting could not be assumed. It also decreased the overall length and redundancy of the procedure for a within-participant procedure (e.g., Rachlin \& Jones, 2008).

\section{Demographic Questionnaire}

After completing all three discounting measures, participants self-reported demographic measures such as age, height, weight, gender, ethnicity, income, and licit/illicit drug use. Demographic questions were always presented in the same order.

\section{Analyses}

Data fidelity were assessed in two steps for monetary and personal information social discounting tasks. Step 1 included eliminating participants that took too long to complete all three measures (i.e., univariate time outliers via Grubbs' test; Grubbs, 1969), and Step 2 included identifying participants who either did not discount, or discounted nonsystemically (Johnson \& Bickel, 2008). Nondiscounters were excluded because their choices showed insensitivity to changing social distances, whereas nonsystematic discounters were excluded because their choices indicated poor stimulus control. Examples of a participants that met the Step 2 exclusion criteria would be someone who always (or never) shared at each social distance, or someone whose indifference point at social distance \#1 was $\$ 80$ (only shared), \$50 at social distance \#2, and \$80 once again at social distance \#5. One participant was a time outlier, and 23 participants either did not 
Table 1 Demographic Information for Participants Based on Exclusion Criteria

\begin{tabular}{llll}
\hline & & \multicolumn{2}{l}{ Analyzed Sample } \\
\cline { 3 - 4 } & Full Sample & Money & Personal Information \\
\hline $\mathrm{n}=$ & 96 & 72 & 88 \\
Mean $(S D):$ & & $18.5(0.9)$ & $18.5(0.9)$ \\
Age & $18.5(0.9)$ & $66.9(4.2)$ & $67.1(4.2)$ \\
Height (inches) & $67.3(4.8)$ & $143.4(27.9)$ & $147.1(31.3)$ \\
Weight (pounds) & $146.3(31.0)$ & & \\
\%: & & 75.0 & 72.7 \\
Female & 71.9 & 30.6 & 27.3 \\
Non-White & 28.1 & 26.4 & 28.4 \\
Income < \$25,000/year & 28.1 & 45.8 & 44.3 \\
Drink Alcohol & 41.7 & 19.4 & 19.3 \\
Use Marijuana & 18.8 & & \\
Mean $s$-value (median)/VAC*: & & $0.28(0.03) / 0.74$ & $0.52(0.03) / 0.72$ \\
Standard Magnitude \$ & $0.49(0.04) / 0.71$ & $0.50(0.05) / 0.74$ & $0.72(0.05) / 0.73$ \\
Large Magnitude \$ & $0.68(0.04) / 0.73$ & $0.27(0.11) / 0.83$ & $0.29(0.10) / 0.84$ \\
Personal Information & $0.27(0.09) / 0.82$ & &
\end{tabular}

*s-values \& VAC could only be calculated for participants that discounted discount hypothetical money as a function of social distance, or increased the amount shared by more than $20 \%$ from the previous social distance on at least one occasion, for either the standard or large magnitude social discounting task. Thus, 72 participants' data were analyzable for the monetary social discounting tasks. For personal information social discounting, seven participants either did not discount personal information as a function of social distance, or increased the amount shared by more than $20 \%$ from the previous social distance on at least one occasion. Thus, 88 participants' data were analyzable for the personal information discounting task. Sixty-four participants completed all three discounting tasks successfully.

Discounting rates were calculated using a hyperbolic function (Jones \& Rachlin, 2006):

$V=\frac{U}{(1+s N)}$

where $V$ is the subjective value for the commodity, $U$ is the objective unshared commodity at social distance $=0$, and $N$ is the social distance between the participant and individual with whom the sharing could occur. The $s$ parameter is a constant representing social distance sensitivity, where larger $s$-values indicate less sharing (i.e., more selfishness) as social distance increases. Discounting rates and variance accounted for (VAC) for each participant were estimated using nonlinear regression fit to the above hyperbolic function (Reed et al., 2012) using Microsoft Excel, 2019 version.

To test whether individuals also share personal information like hypothetical money as social distance increases, Pearson product-moment correlation coefficients were calculated between $s$-values for both hypothetical monetary magnitudes and personal information. In addition, a repeated measure ANOVA was performed with $s$-values being the repeated measure. Follow-up dependent $t$-tests were conducted to determine significant differences between $s$-values. The follow-up dependent $t$-tests also tested any potential magnitude effect (i.e., Hypothesis 2) more directly.

\section{Results}

Descriptive demographic information is shown in Table 1 as a function of exclusion criteria. Participants were mostly white females with incomes $>\$ 25,000 /$ year. Fewer than half of the participants reported drinking alcohol, and between $18.8 \%-19.4 \%$ reported current marijuana use depending on the analyzed sample. Demographic data did not significantly change as a function of exclusion criteria for either monetary or personal information social discounting. KolmogorovSmirnov normality tests showed that $s$-values were not normally distributed for any social discounting tasks (all $D$ 's $>0.37$; $p$ 's $<0.001$; see also Table 1 means vs. medians). However, a logarithmic transformation (base 10) resulted in normally distributed values in all cases (all $D$ 's $<0.12 ; p$ 's $>$ $0.1)$. Thus, logarithmically transformed $s$-values were used in all subsequent analyses.

The $\log s$-values for the standard and large magnitude monetary discounting were significantly positively correlated, $r(62)=0.68, p<0.001$. However, personal information $\log s$-values were not significantly positively correlated to either the standard magnitude monetary log 
Table 2 Pearson Correlation Coefficients (r) at each Social Distance between Tasks

\begin{tabular}{llllllll}
\hline \multicolumn{7}{l}{ Social Distance } \\
\cline { 2 - 7 } & 1 & 2 & 5 & 10 & 20 & 50 & 100 \\
\hline PI \& SM & 0.078 & 0.100 & 0.208 & 0.298 & 0.373 & 0.304 & \\
PI \& LM & 0.143 & 0.256 & 0.175 & 0.309 & 0.282 & 0.121 & \\
SM \& LM & 0.299 & 0.474 & 0.536 & 0.638 & 0.612 & 0.687 & 0.758 \\
& PI = Personal Information & & & & \\
& SM = Standard Magnitude Money & & & & \\
& LM = Large Magnitude Money & & & & & \\
\end{tabular}

$s$-values $(r(62)=0.23, p=0.06)$ or large magnitude log $s$-values $(r(62)=0.22, p=0.08)$. Because there were an unequal number of social distances between the personal information and monetary social discounting tasks, correlations at each social distance between tasks were also calculated. Table 2 shows those correlation coefficients. In general, correlation coefficients increased between measures as social distance increased, except for correlations between personal information and large magnitude social discounting. It should be noted that the largest correlation coefficients were found between the standard and large magnitude social discounting, with all but one correlation coefficient $>0.45$. On the other hand, no correlation coefficient between personal information social discounting and social discounting for monetary rewards was > 0.38 . Table 1 shows that mean variance accounted for (VAC) when Equation 1 was fit to each participant's data was moderate $(>0.70)$ for the two hypothetical monetary social discounting tasks, and good $(>0.80)$ for the personal information discounting tasks. VAC was normally distributed across participants, as mean VAC approximated median VAC.

Figure 1 shows the mean percentage shared for each commodity, along with best-fit hyperbolic curves for each commodity, based on Equation 1. Means are based on all analyzable participants for each commodity, which are different between commodities (see Analyses section). Mean and median $s$-values are shown in Table 1. The figure shows that both magnitudes for hypothetical money and personal information approximated hyperbolic discounting. Personal information showed the steepest discounting as a function of social distance, whereas discounting for the large magnitude money was steeper than that for the standard magnitude money. A repeated measure ANOVA showed a statistically significant difference between $\log s$-values for the 64 participants that completed all three discounting measures, $F(62)=$ $11.22, p<0.001$. Follow-up $t$-tests for $\log s$-values showed a magnitude effect where $\log s$-values for the standard magnitude were significantly smaller than the large magnitude $t(62)=2.23, p=0.03, d=0.28$. Likewise, personal information $\log s$-values were significantly larger than both the standard magnitude $(t[62]=4.26, p<0.001, d=0.53)$ and large magnitude $\log s$-values $(t[62]=2.71, p<0.01, d=$ $0.34)$

\section{Discussion}

Previous social discounting studies have only focused on money as the sharable commodity, showing that the amount shared decreases hyperbolically as a function of social distance (Jones \& Rachlin, 2006). The current study showed that the percentage of personal information shared also decreases hyperbolically as a function of social distance, albeit at a quicker rate (see Figure 1). However, there were no significant correlations between sharing rates for hypothetical money and personal information. This suggests that



Figure 1 Mean Percent of Each Hypothetical Commodity Shared as a Function of Social Distance. Note. Standard magnitude (\$80) hypothetical money, large magnitude (\$800) hypothetical money, and personal information are represented by filled circles, open circles, and grey triangles, respectively. Vertical error bars represent the standard error of the mean. Black, dashed, and grey lines represent best-fit lines for standard magnitude, large magnitude, and personal information, respectively. Best fit lines are based on Equation 1 using mean indifference points. 
social discounting rates for money are relatively independent from those for personal information, like previous delay discounting commodity effects (Charlton \& Fantino, 2008; Weatherly et al., 2010). A social discounting magnitude effect was also shown using a within-participant design and U.S. participants, replicating studies that have shown this effect with larger magnitude differences in different countries (Osinksi, 2010; Rachlin \& Jones, 2008; Romanowich \& Igaki, 2017).

Participants shared personal information as a function of social distance in a hyperbolic manner consistent with previous social and delay discounting studies for hypothetical monetary rewards. However, there were two differences for sharing personal information relative to money. First, personal information discounting rates were significantly steeper than both monetary magnitudes. For example, Figure 1 shows that at social distance 5 the mean percentage shared was $65 \%$ for personal information, compared to $82 \%$ and $78 \%$ for the standard and large monetary tasks (median percentage showed the same pattern; $70 \%$ for personal information vs. $100 \%$ for both monetary tasks). This suggests that participants were more cautious with sharing personal information, relative to money, even at close social distances. Consistent with the magnitude effect, another way to interpret this finding is that personal information is more valuable than money for most participants, which results in less sharing. Second, VAC was larger for sharing personal information, relative to both money magnitudes (see Table 1). All but eight (8.3\% total sample) participants systematically discounted personal information as a function of social distance, and only two participants did not discount sharing personal information (one shared at all social distances; one never shared). By contrast, 24 (25\% total sample) different individuals either did not systematically discounting sharing monetary rewards or did not discount sharing monetary rewards (only sharing at all social distances). This percentage for nonsystematic/nondiscounters is similar to previous hypothetical monetary social discounting studies (i.e., Romanowich et al., 2021). Taken together, this suggests that, in general, participants were more sensitive to sharing personal information as a function of social distance, relative to sharing money.

Participants also showed a magnitude effect, whereby less sharing for monetary rewards occurred when reward magnitude increased. One previous study using relatively smaller magnitude differences (10x) found a magnitude effect for Japanese participants, but not U.S. participants (Romanowich \& Igaki, 2017). Previous studies using larger magnitudes (100x and more) have reported a magnitude effect for social discounting (Osinksi, 2010; Rachlin \& Jones, 2008). Unlike the study by Romanowich and Igaki (2017) the current study used a within-participant design, which increased statistical power (Charness et al., 2012). Even so, the effect size for the current magnitude difference was relatively small $(d=0.28)$. Thus, it is less surprising that Romanowich and Igaki (2017) only found a magnitude effect for the Japanese sample, which contained 79 more participants than the U.S. sample. A within-participant design also adds to the external validity for sharing as a function of magnitude. That is, individuals are likely to make many choices throughout their life about whether to share or not for various monetary magnitudes. Future social discounting magnitude effect studies could profitably focus on parametrically assessing the point at which the magnitude effect disappears using within-participant designs. That is, at what point is the magnitude difference too small to emit a sharing difference.

The results for the personal information social discounting task provide evidence for individuals involved in scams to mimic individuals at closer social distances. Whereas the propensity to share money is still high $(68 \%-70 \%)$ at social distance 10 , on average less than $50 \%$ of personal information was shared at social distance 10 (see Figure 1). Thus, depending on what commodity the scammer is trying to steal, different social distances ranges may yield different success rates. However, this assumes that the person being scammed believes that they are being contacted by someone at a close social distance. It is plausible that discriminating between the real individual and the scammer at close social distances ( 1 or 2$)$ will be easier. However, this hypothesis has not yet been tested. Future personal information social discounting research should focus on this as well as whether those individuals who are more likely to share personal information are also more likely to be the victim of a scam involving personal information. This would test whether personal information social discounting also has predictive validity. Delay discounting has shown significant associations between discounting rates and actual health behaviors (e.g., substance use disorders; MacKillop et al., 2011), as have social discounting studies (e.g., texting while driving; Romanowich et al., 2021). More domain-specific discounting tasks are likely to show stronger associations with these behaviors in the same domain (Lawyer \& Schoepflin, 2013), and vice versa.

The current study has some limitations that should be considered. First, the monetary and personal information social discounting tasks were not symmetrical. The personal information social discounting task did not include social distance 100 and was based on a percentage of sharable information, rather than an absolute amount. It is unknown whether framing personal information as a percentage instead of an absolute amount caused the significantly steeper discounting rate observed for sharing personal information. However, hyperbolic discounting was still approximated in all cases, suggesting that both approaches (percentage and absolute amount) are valid ways to assess social discounting. In addition, previous studies assessing 
magnitude effects have used fewer social distances, with similar results (Rachlin \& Jones, 2008). Future studies should test whether discounting rates systematically change as a function of how the sharable commodity is described (percentage or absolute amount). Second, and related to the first limitation, the personal information social discounting task may be more similar to a social discounting task where the person sharing can keep either everything or nothing (i.e., altruism; Rachlin \& Jones, 2008). It is currently unknown how similar the altruism social discounting task is to the social discounting task for monetary rewards used in the current study. If there is little relationship between those two social discounting tasks for monetary rewards, this may explain the lack of a correlation between the personal information social discounting task and the social discounting tasks for monetary rewards outside of any commodity effect. Lastly, both commodities were hypothetical. Previous social discounting studies have shown that social discounting rates are comparable for hypothetical and real money (Locey et al., 2011). Differences between hypothetical and real personal information should also be tested, because this is critical for assessing whether individuals that share more personal information during a social discounting task will also be more susceptible to scams seeking sensitive personal information.

\section{Conclusion}

The current study is the first to show that sharing a nonmonetary commodity decreases hyperbolically as a function of social distance. In this case, sharing personal information exhibited similar discounting properties to monetary rewards. In addition, a magnitude effect was observed at a relatively small magnitude difference using a within-participant design for the first time with U.S. participants. Given that most participants were still willing to share some personal information with individuals at social distances $>5$, future research should examine what type of personal information is most likely to be shared, and whether those participants who choose to share more personal information also are at greater risk for scams targeting personal information.

Availability of Data and Material The datasets generated during and/or analyzed during the current study are available from the corresponding author on reasonable request.

Authors' Contributions The first author conceived of the study, carried out IRB submission/approval, data collection and analysis, and wrote the entire manuscript.

Funding Data collection and manuscript preparation was partially funded by a Center for Undergraduate Research \& Creative Inquiry (CURCI) internal grant to the first author.

\section{Declarations}

Conflicts of Interest/Competing Interests Not applicable.

Consent to Participate All participants consented to the study protocol before study initiation.

Ethics Approval Approval was obtained from the ethics committee at Gonzaga University (protocol \#2002ROMPSY, Romanowich). The procedures used in this study adhere to the tenets of the Declaration of Helsinki.

Code Availability Not applicable.

Consent for Publication Not applicable.

Open Access This article is licensed under a Creative Commons Attribution 4.0 International License, which permits use, sharing, adaptation, distribution and reproduction in any medium or format, as long as you give appropriate credit to the original author(s) and the source, provide a link to the Creative Commons licence, and indicate if changes were made. The images or other third party material in this article are included in the article's Creative Commons licence, unless indicated otherwise in a credit line to the material. If material is not included in the article's Creative Commons licence and your intended use is not permitted by statutory regulation or exceeds the permitted use, you will need to obtain permission directly from the copyright holder. To view a copy of this licence, visit http://creativecommons.org/licenses/by/4.0/.

\section{References}

Brasor, P. (2019). NHK docudrama reveals telephone scam tactics. Japan Times. https://www.japantimes.co.jp/news/2019/04/20/ national/media-national/nhk-docudrama-reveals-telephone-scamtactics/

Buddiga, N. R., \& Locey, M. L. (2021). Social discounting towards relatives and nonrelatives. The Psychological Record. https://doi. org/10.1007/s40732-021-00479-6

Charlton, S. R., \& Fantino, E. (2008). Commodity specific rates of temporal discounting: Does metabolic function underlie differences in rates of discounting? Behavioural Processes, 77, 334-342.

Charness, G., Gneezy, U., \& Kuhn, M. A. (2012). Experimental methods: Between-subject and within-subject design. Journal of Economic Behavior \& Organization, 81(1), 1-8.

Coombs, C., Coombs, L., \& Lingoes, J. (1978). Stochastic cumulative scales. In S. Shye (Ed.), Theory construction and data analysis in the behavioral sciences (pp. 280-298). Jossey-Bass.

Grubbs, F. E. (1969). Procedures for detecting outlying observations in samples. Technometrics, 11(1), 1-21.

Johnson, M. W., \& Bickel, W. K. (2008). An algorithm for identifying nonsystematic delay-discounting data. Experimental \& Clinical Psychopharmacology, 16, 264-274.

Jones, B., \& Rachlin, H. (2006). Social discounting. Psychological Science, 17(4), 283-286.

Lawyer, S. R., \& Schoepflin, F. J. (2013). Predicting domain-specific outcomes using delay and probability discounting for sexual versus monetary outcomes. Behavioural Processes, 96, 71-78.

Locey, M. L., Jones, B. A., \& Rachlin, H. (2011). Real and hypothetical rewards in self-control and social discounting. Judgement \& Decision Making, 6(6), 522-564. 
MacKillop, J., Amlung, M. T., Few, L. R., Ray, L. A., Sweet, L. H., \& Munafò, M. R. (2011). Delayed reward discounting and addictive behavior: A meta-analysis. Psychopharmacology, 216(3), 305-321.

Martens, M., De Wolf, R., \& De Marez, L. (2019). Investigating and comparing the predictors of the intention towards taking security measures against malware, scams and cybercrime in general. Computers in Human Behavior, 92, 139-150.

Matsumoto, D., \& Juang, L. (2017). Culture and psychology. Cengage Learning.

Mazur, J. E. (1987). An adjusting procedure for studying delayed reinforcement. In M. L. Commons, J. E. Mazur, J. A. Nevin, \& H. Rachlin (Eds.), Quantitative analyses of behavior: Vol. 5. The effect of delay and intervening events on reinforcement value ( $\mathrm{pp}$. 55-73). Lawrence Erlbaum Associates.

Odum, A. L., Becker, R. J., Haynes, J. M., Galizio, A., Frye, C. C. J., Downey, H., Friedel, J. E., \& Perez, D. M. (2020). Delay discounting of different commodities: Review and theory. Journal of the Experimental Analysis of Behavior, 113(3), 657-679.

Osinksi, J. (2010). Social discounting: the effect of outcome uncertainty. Behavioural Processes, 85, 24-27.

Rachlin, H., \& Jones, B. A. (2008). Social discounting and delay discounting. Journal of Behavioral Decision Making, 21(1), $29-43$.

Reed, D. D., Kaplan, B. A., \& Brewer, A. T. (2012). A tutorial on the use of Excel 2010 and Excel for MAC 2011 for conducting delaydiscounting analyses. Journal of Applied Behavior Analysis, 45, 375-386.

Romanowich, P., \& Igaki, T. (2017). Effects of reward magnitude, tobacco, and alcohol use on social discounting in Japan and
United States college students. The Psychological Record, 67, 345-353.

Romanowich, P., Igaki, T., Yamagishi, N., \& Norman, T. (2021). Differential associations between risky cell-phone behaviors and discounting types. The Psychological Record, 71(2), 199209. https://doi.org/10.1007/s40732-020-00446-7

Rosoff, S. M., \& Pontell, H. N. (2011). Cybercrime. In C. D. Bryant (Ed.), Routledge handbook of deviant behavior (pp. 417-426). Routledge. https://doi.org/10.4324/9780203880548

Stegall, S. E., Collette, T., Kinjo, T., Takahashi, T., \& Romanowich, P. (2019). Quantitative cross-cultural similarities and differences in social discounting for gains and losses. Frontiers in Public Health-Health Economics. https://doi.org/10.3389/fpubh.2019. 00297

Weatherly, J. N., Terrell, H. K., \& Derenne, A. (2010). Delay discounting of different commodities. Journal of General Psychology, 137, 273-286.

The author thanks Anna Gade for her help during data collection. Data collection and manuscript preparation was partially funded by a Center for Undergraduate Research \& Creative Inquiry (CURCI) internal grant from Gonzaga University.

Publisher's Note Springer Nature remains neutral with regard to jurisdictional claims in published maps and institutional affiliations. 